\title{
Leonardo Sciascia et l'esprit des Lumières
}

\section{Raymond Trousson}

\section{(2) OpenEdition}

\section{Journals}

\section{Édition électronique}

URL : http://journals.openedition.org/studifrancesi/9093

DOI : ERREUR PDO dans /localdata/www-bin/Core/Core/Db/Db.class.php L.34 : SQLSTATE[HYO00]

[2006] MySQL server has gone away

ISSN : 2421-5856

\section{Éditeur}

Rosenberg \& Sellier

\section{Édition imprimée}

Date de publication : 1 juin 2008

Pagination : 80-92

ISSN : 0039-2944

\section{Référence électronique}

Raymond Trousson, «Leonardo Sciascia et l'esprit des Lumières », Studi Francesi [En ligne], 154 (LII I I) | 2008, mis en ligne le 30 novembre 2015, consulté le 08 janvier 2021. URL : http:// journals.openedition.org/studifrancesi/9093; DOI : https://doi.org/10.4000/studifrancesi.9093

\section{(ब) $(\Theta \Theta$}

Studi Francesi è distribuita con Licenza Creative Commons Attribuzione - Non commerciale - Non opere derivate 4.0 Internazionale. 


\section{Leonardo Sciascia et l'esprit des Lumières}

Né le 8 janvier 1921 à Racalmuto, près d'Agrigente, fils d'un petit employé des soufrières, Leonardo Sciascia n'en a pas moins eu un contact précoce avec la culture française et l'on relève, éparses dans son œuvre, nombre d'allusions à La Rochefoucauld, Pascal, Courier, Casanova, Sade, Chateaubriand, Hugo, Flaubert, Stendhal, Mallarmé ou Gide. Particulièrement nette peut-être, l'imprégnation de la littérature des Lumières avec laquelle il se sent une véritable connivence, complaisamment confessée dans une interview:

Personalmente posso dirLe che mi sono trovato tra le mani Diderot e Courier prima di Pinocchio e del Cuore; non perché fossi un ragazzo prodigio, ma perché questi (oltre il Telemaco di Fénelon, le novelle del Casti, I promessi sposi e i romanzi storici popolari di Luigi Natoli) erano i soli libri di cui potevo disporre. Che poi Diderot e Courier si trovassero tra i pochi libri di una famiglia di piccoli impiegati e maestri delle elementari, può anche essere indicazione di ordine generale ${ }^{1}$.

Le XVIII ${ }^{\mathrm{e}}$ siècle - à ses yeux «il secolo educatore» ${ }^{2}$ - s'étend pour lui de 1679 à 1814 , de la mort du cardinal de Retz à celle du prince de Ligne, «conosciuto in Sicilia per un suo antenato che ne fu vicerè» (p. 1007), parce que ces deux hommes, en dépit de tout ce qui sépare le prince de l'Eglise du prince wallon, lui semblent faire partie d'une même époque, car Retz avait «una mente settecentesca [...] in corpo seicentesco» (p. 1008). Siècle novateur, caractérisé par son aspiration à la liberté: «la sensibilità, la vivacità, la luminosità» en font «un secolo femminile e, senza alcuna teoria o polemica, come naturalmente, femminista» (p. 1012) où, jusque dans la littérature érotique, l'homme et la femme sont partenaires égaux. Siècle de progrès, freiné par les obscurantismes de tous bords, mais éliminant petit à petit les séquelles du passé: «La maggior parte degli uomini continuava a faticare duramente, a guerreggiare, a conculcare e rapinare i deboli, ad assistere a tremendi spettacoli di «atti di fede» e di giustizie feudali: ma l'idea che i deboli fossero buoni, le guerre stupide, gli «atti di fede» mostruosi, le giustizie feudali ingiuste, e che alla dura fatica dovesse corrispondere il godimento del frutto, si faceva strada. Al centro del secolo, come un sole allo zenit, stanno - 1759, 1763, 1764 - il Candide et il Traité sur la tolérance di Voltaire, il Dei delitti e delle pene di Beccaria» (p. 1012). Quoi de surprenant si ce siècle est celui de quelques figures libératrices - Casanova ou Sade ${ }^{3}$-, et d'une trinité universellement fameuse, même si Sciascia ne rend pas le même culte à chacune de ses personnes.

(1) Cité par G.-P. Prandstraller, Il neo-illuminismo di Sciascia, dans A. MotTA, Leonardo Sciascia: la verità, l'aspra verità, Manduria, Lacaita, 1985, p. 175 .

(2) Cruciverba, dans L. Sciascia, Opere 1971-
1983, a cura di C. Ambroise, Milano, Bompiani, 1989, pp.1006-1016.

(3) Voir, dans Cruciverba, les articles L'Utopia di Casanova (pp. 1017-1025), Casanova o la dissipazione (pp. 1026-1031). 
Rousseau est assurément la moins vénérée, surtout lorsqu'il le compare à son grand contemporain. Instituteur pendant plusieurs années, Sciascia s'est naturellement intéressé à son système pédagogique qui lui paraît absurde, sans prise sur la vie réelle et jamais il n'a pu lire l'Émile «senza irritazione». C'est au point que la fameuse lettre, qu'il connaît «a memoria», adressée le 30 août 1755 par Voltaire à Rousseau - «J'ai reçu, Monsieur, votre nouveau livre contre le genre humain...»-lui fait éprouver «una gioia incontenibile» ${ }^{4}$. L'œuvre autobiographique n'échappe pas à sa critique impitoyable: aux Confessions rousseauistes, l'écrivain assure préférer celles de saint Augustin ${ }^{5}$. Ses propos ne sont pas plus amènes dans une interview, où il tente de s'expliquer à lui-même les raisons de cette antipathie profonde pour l'idéologue naufrageur du Contrat social:

In realtà, Rousseau non m'interessa affatto. L'Emilio, che ho letto all' età di quattordici anni, mi è parso un libro fondamentalmente falso. E in quanto a confessioni, preferisco quelle di Sant'Agostino. E poi: la volontà generale! Con tutti gli ismi che vengono da lì. Mentre la democrazia è l'espressione di una volontà aritmetica, quella della maggioranza, Rousseau ha creduto di scoprire una «volontà generale» che non coincide con la legge del numero massimo, che può essere l'appannaggio di pochi o di qualcuno, pur pretendendo di imporsi come interprete della volontà di tutti. Proclamando che la parte può sostituire il tutto, parlando di volontà generale, Rousseau è all'origine dei principali mali del nostro tempo ${ }^{6}$.

Jugement sévère. Ce qui gêne Sciascia chez Jean-Jacques, c'est la rigidité et l'intransigeance de sa pensée, surtout en politique. Il est de ceux qui tiennent ses théories pour néfastes à tout esprit de liberté. Rousseau est l'ancêtre de la démocratie totalitaire, le père lointain du fascisme. Si Voltaire enseigne la liberté, Jean-Jacques mène à la dictature, jacobine ou stalinienne:

Niente ebbero in comune Voltaire e Rousseau. Nemmeno la reciproca disistima. Voltaire aveva per Rousseau un dileggiante disprezzo. Rousseau odiava Voltaire nella misura in cui avrebbe voluto amarlo. Ed erano diversi per estrazione sociale, carattere, idee, modo di vita. L'unico legame tra loro è questo su cui noi oggi ci chiniamo: l'anno della morte. [...] Ma questo solo legame stabilito dalla sorte - e cioè dalla morte - è quel che occorre a noi, in questo 1978, per fare, come si dice in marina, il punto: di dove ci troviamo, della rotta da lasciare e di quella da intrapendere. La rotta Rousseau. La rotta Voltaire. E a prima vista la rotta Rousseau sembra la più vasta, la più sconfinata, la più promettente; e certamente la più affascinante. Ma non è quella che è stata percorsa già? Non è quella del romanticismo, della «volontà generale», della parte maggiore del tutto, degli «ismi» più micidiali?

Cerchiamo di far bene i conti. E anche se di Voltaire non ci resta molto, anche se soltanto ci restano i racconti, le pagine sul caso Calas, un mucchietto di lettere, questo poco teniamocelo, a questo poco afferriamoci. O andremo a fondo con Le Confessioni e l'Emilio appesi al collo ${ }^{7}$.

Tôt découvert, Diderot a droit à un tout autre accueil. Sciascia adolescent l'a abordé dans le Paradoxe sur le comédien, publié dans une collection italienne popu-

(4) L. SCIASCIA, La Sicilia come metafora. Intervista con M. Padovani, Milano, Mondadori, 1979, p. 10 .

(5) Rousseau lui apparaît comme appartenant au XIX ${ }^{\mathrm{e}}$ siècle bien plus qu'au XVIII', ce qui explique qu'il ait indisposé tant de ses contemporains et se soit brouillé avec tous ses amis: «La spiegazione vera sta nell'estraneità di Rousseau, nella sua imprudenza, nel suo essere «postumo» rispetto al se- colo. Rousseau, dice Cocteau, ha commesso la peggiore delle imprudenze: ha reso di pubblica ragione l'intera sua vita. Nell'impero della finzione, in un secolo educatore, in un secolo «vestito», ha commesso l'imprudenza e la scorrettezza di denudarsi. Esibizionisticamente» (Cruciverba, p. 1014).

(6) L. SCIASCIA, La Sicilia come metafora, pp. 57-58. 800 . 
laire: «La prima opera francese a capitarmi tra le mani è stata Il paradosso dell' attore di Diderot, pubblicata in una collana popolare dell'editore Sonzogno, un libro che ho letto senza possedere una grande conoscenza del mondo del teatro e degli attori. In esso, ad affascinarmi fu ben altro: il modo con cui era scritto, l'intelligenza del discorso. Il modo di argomentare» ${ }^{8}$. Il se plaît à rappeler qu'un critique, un jour, l'a rapproché du Langrois: «Non si rendeva conto di avermi fatto il massimo complimento. Diderot: ecco uno scrittore che non smette di crescere e che meriterebbe di esser più amato, persino in Francia, dove non è ancora abbastanza apprezzato» ${ }^{9}$. Comme les marxistes, il salue celui qui a réhabilité les arts mécaniques et élevé le monument encyclopédique à la gloire de la raison et du progrès:

D'altronde, presentendo quest'incomprensione, lui stesso aveva annunciato, nella sua lettera «a coloro che verranno», che sarebbe stato capito solo dai posteri. A parer mio, finirà per risultare più importante di Voltaire ${ }^{10}$.

Dans son journal, Sciascia étend son admiration à ces Bijoux indiscrets si souvent tenus pour œuvre mineure, voire scandaleuse. Lui préfère y voir une bouffonnerie pleine de verve et de sens critique, l'une des voies obliques empruntées par la littérature du temps, qu'il rapproche de la mystification qui a donné naissance à $L a$ Religieuse: «E considerando che anche La Religieuse gli era venuta come uno scherzo, una beffa, une buffoneria [...], possiamo mettere a conto del suo genio l'invenzione dello scherzo come categoria letteraria. [...] Non ci resta che lo scherzo, se vogliamo salvarci; se vogliamo, cioè, salvare l'intelligenza delle cose, dei fatti. Lo scherzo dentro di noi, dentro le cose, i fatti, le idee» ${ }^{11}$. On comprend qu'au cours d'un voyage en France, Sciascia ait tenu à s'arrêter à Langres et à se recueillir devant la statue du philosophe: «Tra Hugo e Diderot oggi la Francia splende nel nostro cuore, è la Francia della nostra storia umana, la Francia dei nostri libri, della nostra ragione. Di solito odiamo i monumenti, ma questo di Diderot nella piazza di Langres, resta come un bellissimo incontro» ${ }^{12}$. C'est sans doute dans ses Cruciverba que le Sicilien a rendu au Langrois l'hommage le plus fervent en saluant celui qui, tout au contraire de JeanJacques, fut de son siècle au point de le représenter dans ses aspirations les plus modernes, du roman à la critique d'art en passant par la théorie du théâtre:

Diderot è la chiave del secolo. Quest'uomo che voleva esser nulla, «ma nulla del tutto», ha come inventato il secolo in quel che noi riconosciamo di più proprio, di più originale, di irripetibile. [...] Ha inventato una professione: la più libera che si potesse immaginare - e per non averne alcuna. E da questa sua professione, da questa sua non-professione, è venuta l'Enciclopedia. E dall'Enciclopedia una nuova concezione del fare, delle attività umane, del lavoro. Un fare che somigliava al non-fare. Un «fare con gioia». Un'utopia, se si vuole. Senz'altro un'utopia. Ne vediamo la rovina, ma ancora la si persegue.

[...] L'Enciclopedia è appunto il tentativo di dare agli uomini la gioia del proprio lavoro: la gioia della conoscenza, dell'intelligenza, dell'armonia delle parti nel tutto. [...] Per non avere alcuna, Diderot ha dunque inventato una professione: quella dell'intellettuale. Nonostante le difficoltà, i pericoli, il carcere, i bisogni, è da credere l'abbia esercitata con gioia. Prendeva tutto sul serio ma con tanta leggerezza da dare l'impressione che non si prendesse sul serio. Scrive La Monaca per fare uno scherzo e I Gioielli indiscreti come per scommessa e per dare del denaro a una donna che ne ha bisogno. Non si cura di dare alle stampe tutto quello che scrive,

(8) L. Sciascia, La Sicilia come metafora, pp. 5657.

(9) L. SCIASCIA, ibid., p. 57.

(10) L. ScIASCIA, ibid., p. 57.
(11) Nero su nero, p. 784.

(12) Cité par M. Collura, Il Maestro di Regalpetra. Vita di Leonardo Sciascia, Milano, Longanesi, 1996, p. 142. 
e anzi ne dà pochissimo; ma nulla di ciò che ha scritto è «postumo» se non accidentalmente. Sta dentro il suo secolo come ogni uomo nella propria pelle. Eppure è sopratutto attraverso la sua opera che il secolo XVIII ci raggiunge, ci occupa, ci offre strumenti e misure. [...] Grande educatore in un secolo educatore (pp. 1015-1016).

$\mathrm{Ni}$ «posthume», ni obsolète, Diderot est de ceux dont le message, aujourd'hui encore, demeure d'actualité parce qu'il a fait confiance à la raison, au savoir, à la science pour affranchir l'homme de l'obscurantisme et des superstitions, du despotisme et de l'Église.

Le XVIII 'e siècle sert de toile de fond au Consiglio d'Egitto, publié en 1963, l'un des romans les plus réussis de Sciascia, dont l'action débute dans la Sicile arriérée de 1782. C'est l'époque où l'île a pour vice-roi un homme des Lumières, le marquis napolitain Domenico Caraccioli, qui avait passé dix ans à Paris. Littérateur de modeste envergure, mais auteur de deux textes qui eurent du succès et en disent long sur son amour de la France des Lumières - L'Europe française (1776) et Paris, le modèle des nations étrangères (1777) -, et dont la première mesure, lorsqu'il débarqua en Sicile en 1781, fut d'abolir la torture. Aux yeux de l'avocat «progressiste» Di Blasi, il est celui qui a entendu la parole des grands réformateurs du siècle: «Ogni volta che lo avvicino, ogni volta che mi rivolge la parola, mi sento... emozionato, ecco, commosso... Quest'uomo, mi dico, ha conosciuto Rousseau, ha conversato con Voltaire, con Diderot, con d'Alembert... A proposito: sapete che Diderot è morto? Il trentuno del mese scorso...» $\gg^{13}$. Le vice-roi s'est empressé, au grand scandale des conservateurs, d'effacer les traces les plus apparentes de la domination cléricale en faisant gratter sur la façade d'un palais le Deus, judica causam tuam, devise de l'Inquisition (p. 22). Soucieux de faire régner une justice plus équitable, il n'a pas tardé à s'en prendre aux privilèges des nobles qui dédaignent d'honorer leurs dettes, de payer les impôts sur leurs fiefs ou refusent d'être poursuivis pour homicide sur la personne d'un gueux, et à mettre au pas des dignitaires de l'Église et cette manière de leur faire rendre gorge l'a bientôt rendu impopulaire: «Non c'era giorno che non tirasse fuori una nuova angheria, che non cacciasse il suo volteriano naso nelle cose della religione» (pp. 33-34). Lutte inégale des lumières contre l'ombre. Caraccioli n'empêchera pas les fêtes somptueuses en l'honneur de sainte Rosalie et clergé et aristocrates se réjouiront de la victoire remportée sur celui qui «stava col grifo affondato nel truogolo di quel Voltaire» (p. 40). Le marquis succombera devant ceux qui intriguent pour obtenir son rappel. C'est avec un sourire un peu amer qu'il dira à Di Blasi, au moment de quitter l'île, ce mot qui, entre eux, est indice d'une connivence: «Come si può essere siciliani?»»

En face de ce personnage historique, Sciascia a campé deux héros imaginaires. Le premier, l'abbé Vella, aspirant à une existence confortable et à quelque bénéfice ecclésiastique, a monté une extraordinaire imposture, qui consiste à prétendre traduire un manuscrit arabe, simple récit de la vie du Prophète, dont il prétend qu'il est en réalité le Consiglio d'Egitto, qui permettrait de réécrire l'histoire de la Sicile en montrant que les propriétés des grands seigneurs appartiennent en réalité au roi: «Tutto alla Corona, e niente ai baroni». Redoutant ses révélations, les nobles le choient et le couvrent de cadeaux. Vella finit par se prendre à son propre jeu, se complaît dans son rôle comme un acteur, au point d'avoir besoin d'avouer sa mystification, non par remords, mais pour recueillir, fût-ce en courant à sa perte, les applaudissements d'un public: «La sua disperazione d'artista si fondeva a quella vanità comune a tutti gli uomini che delinquono: aveva bisogno di qualcuno, spettatore e complice, che in 
lui ammirasse, nel quotidiano lavoro, l'originale creatore di un'opera letteraria e il non meno originale e spericolato impostore» (p. 63). Le voilà dans la situation d'un comédien fatigué de se répéter - l'occasion, pour Sciascia, de se référer au Paradoxe sur le comédien: «L'abate si sentiva svuotato e stanco come un attore che ha tenuto ruolo principale in una commedia di successo: per sere e sere lo stesso personaggio, la stessa maschera. [...] Anche se fosse stato in voga l'abate avrebbe ritenuto al suo temperamento e al suo caso più adatto il Paradoxe sur le comédien» (p.121). L'Histoire qu'il écrit est fausse? Qu'importe, puisqu'il aura du moins montré les usurpations de la noblesse, la corruption de l'ordre social et que du reste, «la storia non esiste» et que le travail de l'historien est toujours imposture. Aussi Vella, sceptique, ne peut-il imaginer dans l'éventuelle et improbable instauration d'une république en Sicile qu'une autre forme de duperie. Il ne peut cependant se défendre d'admirer la Révolution française: «Per me repubblica e regno sono lo stesso brodo, la stessa soperchieria. [...] Per la rivoluzione, ve lo confesso, ho invece un sentimento diverso: [...] mi piace... I potenti che vanno ad intanarsi e i miseri che fanno trionfo...» (p. 154).

Le second personnage inventé est l'avocat libéral Francesco Paolo Di Blasi, gagné aux principes des Lumières et grand admirateur des philosophes, qui possède dans sa bibliothèque l'Encyclopédie et les œuvres de Diderot dans l'édition de 1773 et prête sous le manteau, à une jolie marquise émancipée, les scandaleux Bijoux indiscrets. Il incarne une jeunesse intellectuelle, ouverte aux idées nouvelles, admiratrice de la Révolution de 1789 et favorable aux réformes audacieuses de Caraccioli. Ennemi de sa propre classe, avec Jean-Jacques il réclame l'égalité, le droit pour le paysan d'être traité comme un homme, l'abolition des privilèges. Idéaliste, il monte un complot destiné à instaurer une république, espérant que les gens des campagnes se dresseront «in nome della fame e delle angherie in cui si dibattevano». Dénoncé, Di Blasi, d'abord atrocement torturé, sera décapité, symbolisant ainsi l'échec de la raison et de la volonté de progrès, la banqueroute de l'enseignement des livres: au moment de son arrestation, il heurte par inadvertance une pile de ses ouvrages chéris qui s'effondre dans un chaos dérisoire. Quel dommage que l'Histoire se confonde - Vella le savait - avec le mensonge, ou que la pensée des grands philosophes, comme leur vie, se soit arrêtée, inefficace, au seuil de la Révolution et que les livres le conduisent seulement, lui, à la mort d'un martyr des Lumières ${ }^{14}$ :

La menzogna è piú forte della verità. Piú forte della vita. Sta alle radici dell'essere, frondeggia al di là della vita.. [...] E crediamo che la verità era prima della storia, e che la storia è menzogna. Invece è la storia che riscatta l'uomo dalla menzogna, lo porta alla verità: gli individui, i popoli...»E a se stesso, con irrisione, con compatimento: «Se hai creduto in Rousseau, è giusto che tu ne veda il contrappasso nell'abate Vella...» Ma ne ebbe smarrimento, come di una bestemmia scaturita da un inciampo improvviso, da un urto imprevedibile. «Il fatto è che Voltaire ti serve di piú, oggi... Ma forse Voltaire serve sempre di piú... Non quanto vorresti, però... Quel che vorresti è il loro pensiero, di Voltaire, di Diderot, e anche di Rousseau, dentro la rivoluzione: e invece si è fermato sulla soglia, come la loro vita... (p. 119).

Pis encore, l'Histoire à venir - heureusement il l'ignore - sera lourde d'injustices et de cruautés, d'autres seront persécutés et torturés comme si jamais n'avaient existé les Lumières et la foi dans le progrès de la raison et de l'humanité. Conclusion singulièrement pessimiste sur un futur où les Di Blasi et les Caraccioli ne sont pas près de l'emporter sur la sauvagerie et la brutalité du pouvoir:

(14) R. Mortier, Préjugés des «philosophes» et message des Lumières, dans Palermo-Paris. Parigi-
Palerme. Due capitali culturali fra il Settecento e il Duemila, Palermo, G.B. Palumbo, 2000, p. 398. 
E la disperazione avrebbe accompagnato le sue ultime ore di vita se soltanto avesse avuto il presentimento che in quell'avvenire che vedeva luminoso popoli interi si sarebbero votati a torturarne altri; che uomini pieni di cultura e di musica, esemplari nell'amore familiare e rispettosi degli animali, avrebbero distrutto milioni di altri esseri umani: con implacabile metodo, con efferata scienza della tortura; e che persino i più diretti eredi della ragione avrebbero riportato la «questione» nel mondo: e non più come elemento del diritto, quale almeno era nel momento in cui lui la subiva, ma addirittura come elemento dell'esistenza (pp. 177-178).

Des allusions significatives au XVIII ${ }^{\mathrm{e}}$ siècle apparaissent aussi, en 1971, dans Il Contesto, qui s'ouvre sur une épigraphe empruntée au livre IV de l'Émile: «O Montaigne! Tu che ti picchi di franchezza e di verità, sii sincero e verace, se può esserlo un filosofo, e dimmi se esiste sulla terra un paese dove sia delitto il mantenere la parole data e l'essere clementi e generosi; dove il buono sia disprezzato e onorato il malvagio». À quoi répond un «anonyme» qui n'est autre que Sciascia lui-même: «O Rousseau! $\gg^{15}$. Si le roman évoque notre société ou plutôt le pouvoir qui la domine, qui de nous, aujourd'hui, ne connaît un pays aussi inhumain?

Il s'agit à première vue d'un roman policier, mais dont l'auteur nous avertit qu'il a voulu réaliser «une parodie». Les ingrédients du genre y sont: le meurtre de plusieurs juges, un assassin nommé Crès et, à ses trousses, le détective Rogas. La finale est moins banale: nul coupable ne finit sous les verrous et Rogas est assassiné à son tour. On s'aperçoit bientôt que le récit débouche, non sur une intrigue policière, mais sur une réflexion désabusée sur la société: «Ho cominciato a scriverla [cette parodie] con divertimento, e l'ho finita che non mi divertivo più» (p. 96). La «nota» accompagnant le roman souligne la dimension politique et morale de ce qui est bel et bien «un apologo sul potere nel mondo»:

Ad un certo punto la storia cominciò a muoversi in un paese del tutto immaginario; un paese dove non avevano più corso le idee, dove i principi - ancora proclamati e conclamati - venivano quotidianamente irrisi, dove le ideologie si riducevano in politica a pure denominazioni nel giuoco delle parti che il potere si assegnava, dove soltanto il potere per il potere contava (p. 95).

Au cours de son enquête, Rogas mène le lecteur dans les milieux de la magistrature, du pouvoir et du monde littéraire, lui-même prisonnier - «in detenzione» - dans un Etat «detenuto», luttant pour au moins «aprire una crepa nel muro» (p. 66). Les magistrats sont corrompus, l'administration de la justice contrecarre l'enquête de Rogas, lui enjoint d'abandonner la piste qu'il croit la bonne pour s'orienter vers les groupuscules révolutionnaires, solution politiquement plus utile. Intègre, Rogas suit son chemin, convaincu que les meurtres sont l'œuvre d'un homme, le pharmacien Crès, victime d'une erreur judiciaire, acharné à se venger d'une justice inique. Lorsqu'il se présente chez Riches, président de la Cour suprême, pour le prévenir que ses jours sont en danger, le magistrat méprise ses avertissements, tout en refusant d'admettre la possibilité même de l'erreur: «L'errore giudiziario non esiste» (p. 70). Infaillible comme un dogme, la justice s'accomplit toujours, comme dans le «mistero della transustanziazione». La croyance néfaste à l'erreur judiciaire, continue le magistrat, est l'œuvre de Voltaire, dont il cite le début du Traité sur la tolérance, où le philosophe explique que si l'on oublie bientôt ceux qui, à la guerre, tombent les armes à la main et ont eu la possibilité de se défendre, «la voce pubblica» réclame vengeance pour un père de famille innocent «caduto nelle mani dell'errore, o della passione, o del fanatismo» (p. 71). 
Là, selon Riches, est la faute de Voltaire. La distinction entre les deux types de mort n'existe pas, car «la giustizia siede su un perenne stato di pericolo, su un perenne stato di guerra», et c'était vrai déjà au XVIII ${ }^{\mathrm{e}}$ siècle, «ma non si vedeva; e comunque Voltaire era troppo grossolano per accorgersene». Les masses elles-mêmes ont «portato l'esistenza umana a un totale e assoluto stato di guerra» (p. 72) et de ce principe découle cette étrange et terrifiante conception de la justice: «La sola forma possibile di giustizia, di amministrazione della giustizia, potrebbe essere, e sarà, quella che nella guerra militare si chiama decimazione. Il singolo risponde dell'umanità. E l'umanità risponde del singolo. Non ci potrà essere altro modo di amministrare la giustizia. Dico di più: non c'è mai stato. Ma ora viene il momento di teorizzarlo, di codificarlo» (p. 72). L'individu et la responsabilité individuelle n'existent pas:

Il problema della giustizia, per Voltaire e per quelli che discendono da lui, sembra incentrarsi su quei delitti che chiama locali. Ma ora la massa, dilagando sui codici come una mandria assetata, assetata di delitto voglio dire, ha cancellato i delitti locali. Il giudice non ha più da domandarsi: «Je n'oserais punir à Ragusa ce que je punis à Lorette». Quel che si punisce a Raguse si punisce a Loreto. Ma meglio sarebbe dire quel che non si punisce... Poche cose si puniscono, ormai (pp. 73-74).

Devant les protestations de Rogas, Riches lui explique que les crimes sont tous universels parce qu'ils sont «delitti contro la leggitimità della forza», parce que la justice est devenue pouvoir et qu'ainsi tout délit est crime de lèse-majesté ${ }^{16}$. Condamné, l'accusé se soumet à la toute-puissance divinisée qui l'écrase: «La confessione di una colpa da parte di chi non l'ha commessa stabilisce quello che io chiamo il circuito dela legittimità» (p. 75). La culpabilité, comme devant le tribunal de l'Inquisition, ne dépend pas des preuves, mais de l'état de culpabilité où est placé l'individu. Il importe donc fort peu que Crès soit en réalité innocent ou coupable: «E dallo stato di colpa è facile estrarre gli elementi della convinzione di reato più che dalle prove oggettive, che non esistono; e anzi, se mai, sono le prove oggettive che possono dar luogo a quello che lei chiama errore giudiziario» (p. 75). Rogas n'en défendra pas moins le principe du Traité voltairien contre le magistrat, «quello che ti blocca a confidarti la sua utopia, la sua civitas dei, il suo falansterio» (p. 72) et exige la soumission de l'éthique au politique. En vain, puisque la finale les renverra dos à dos: Rogas est assassiné par le pouvoir, Riches par Crès. Voltaire ne l'emporte pas dans notre monde. Sciascia le sait, lui qui, dans L'Affaire Moro (1978), dont le titre même fait écho à l'affaire Calas, dénonce la capitulation du gouvernement italien devant le terrorisme des Brigades rouges. Si l'on est loin, dans Il Contesto, du roman historique du Consiglio d'Egitto, l'esprit des Lumières n'y est pas moins présent.

Il l'est encore dans Todo modo (1975), autre roman politique filé sur une trame policière où sont mis en évidence les liens entre l'Église et le pouvoir. Histoire du meurtre de notables au cours des exercices spirituels organisés par don Gaetano, qui sera lui-même assassiné. Ici, un peintre libre penseur affronte le prêtre, nouvelle mouture de l'éternel inquisiteur à la Dostö̈evski, et de nouveau les Lumières sont mises en cause: «Il secolo diciottesimo ci ha fatto perdere il senno, il ventesimo ce lo farà riguadagnare» (p. 186). Il faut détruire le monde, afin que puisse s'y accomplir enfin la venue de Dieu. L'épouvante pascalienne devant l'infini se voit curieusement rapprochée par don Gaetano de la sérénité voltairienne désormais impossible dans un monde décidément bouleversé où la raison n'a plus cours: 
È stato detto che il razionalismo di Voltaire ha uno sfondo teologico incommensurabile all'uomo quanto quello di Pascal. Io direi anche che il candore di Candide vale esattamente quanto lo spavento di Pascal, se non è addirittura la stessa cosa. Solo che Candide trovava finalmente un proprio giardino da coltivare... «Il faut cultiver notre jardin»... Impossibile: c'è stato un grande e definitivo esproprio. E forse si possono oggi riscrivere tutti i libri che sono stati scritti. [...] Tutti. Tranne Candide ${ }^{17}$.

On n'aura pas manqué de l'observer, si Rousseau est condamné comme ancêtre des totalitarismes précisément dénoncés dans Il Contesto ou Todo modo, si Diderot au contraire est célébré, c'est cependant Voltaire qui tient chez Sciascia le premier rang. «Ritengo che Voltaire sia ben piazzato sulla linea d'arrivo - ammesso che essa esista - degli scrittori». Voltaire, dit-il dans une interview, «quest'esempio di professionalità della scrittura, questo modello di scrittore, chiaro, svelto, conciso, intelligente, sintetico, ironico: ecco tutto ciò che per me rappresenta la chiave della scrittura e del vero mestiere ${ }^{18}$. Sciascia vénère l'écrivain, le styliste sans égal, mais n'exalte pas moins le champion de la libre pensée, l'irréductible adversaire de la bêtise et du fanatisme, deux fléaux au moins aussi actifs aujourd'hui que jadis, et il se plaît à s'imaginer fustigeant comme son modèle les sots et les barbares:

Voltaire diceva che la più grande sventura, per lo scrittore, non è quella di essere invidiato dai colleghi, vittima degli intrighi, disprezzato dai potenti, ma di essere giudicato dagli imbecilli, i quali a volte arrivano lontano, surtout quand le fanatisme se joint à l'ineptie. Ma qui bisogna osservare che Voltaire si trovò a vivere in un'epoca in cui non tutti gli stupidi erano fanatici né tutti i fanatici erano stupidi. Oggi la stupidità e il fanatismo sono inseparabili e indistinguibili; non c'è fanatico che non sia stupido, e non c'è stupido che non sia fanatico. [Du moins partage-t-il le plaisir qu'a dû aussi connaître Voltaire] Non è piacere da poco scrivere un libro, un articolo, una nota sapendo già e poi verificandolo negli effetti, che dieci o diecimila fanatici che vi si imbatteranno si sentiranno torcere le viscere, si illividiranno e ci detesteranno ${ }^{19}$.

L'écrivain sicilien ne se plaisait-il pas, lors de ses séjours à Paris, à se faire photogra-

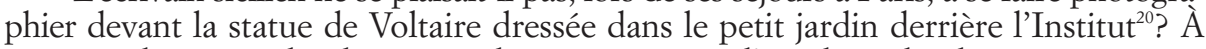
ses yeux, le patriarche de Ferney demeure à jamais l'auxiliaire le plus précieux contre tous les fanatismes, l'ennemi juré d'un clergé qu'aujourd'hui encore il empêche de consolider définitivement son pouvoir. Il le rappelle dans Nero su nero à propos de l'interview d'un prêtre qui, non content d'affirmer sa confiance dans l'avenir de l'Église, s'en prenait à Voltaire:

Ma ecco che, a confermare la sua fede nella divinità della Chiesa, il padre gesuita vien fuori con un esempio che ci riporta ai tempi delle quaresimali superstizioni e maledizioni: «Mi vien alla mente che Voltaire, il 30 maggio 1758, disse: Tra vent'anni il Galileo sarà sbancato. Ebbene: il 20 maggio 1778 Voltaire morì, fu... sbancato lui».

Siamo dunque alle solite. Chi tocca i fili muore. Dio paga vendetta, nonché tutti i giorni, imprevedibilmente, anche a scadenze previste e fisse. Con la sua fida, nel 1758, Voltaire firmò una cambiale che vent'anni dopo, puntualmente sconfitto, doveva pagare con la vita. Ora Voltaire nel 1758 aveva sessantaquattro anni. Si assicurò dunque di vivere per altri venti, raggiungendo gli ottantaquattro: che è una bella età anche ai nostri giorni, cui arridono le magnifiche sorti e progressive della scienza medica. C'è stato poi un anticipo di dieci giorni sulla scadenza: e denuncia una certa impazienza o un errore. Nell'un caso o nell'altro abbiamo, per conto del

(17) Todo modo, dans Opere 1971-1983, pp. 187 188.

(18) L. Sciascia, La Sicilia come metafora, p. 57.

(19) Nero su nero, p. 734.
(20) A. Cavallari, Sciascia all'incrocio con Manzoni («Corriere della Sera», 9 dicembre 1979), dans A. МоттA, Leonardo Sciascia: la verità, l'aspra verità, p. 243. 
signor di Voltaire, dei motivi di appello. A meno che questo scarto di dieci giorni non dimostri appunto il contrario di quel che il padre gesuita vuole suggerire: e cioè che se Voltaire si intrigava nelle cose di Dio, Dio si guardava bene dall'intrigarsi nelle cose di Voltaire (p. 621).

De toutes les œuvres de Voltaire, laquelle avait plus de chances que Candide de retenir l'attention et l'admiration sans réserves de Sciascia? En1983, à l'occasion de la traduction de Bacchelli, chez Einaudi, dans la collection «Scrittori tradotti da scrittori», il accepté d'écrire une brève préface où il souligne l'éternelle actualité du texte:

Lo si legge oggi con lo stesso diletto e forse con più acuta riflessione di come allora fu letto. E possiamo anche azzardare la previsione che quanto più il mondo diventerà irragionevole (e in questo senso velocemente corre) tanto più ragionevoli, con amaro diletto, vi si rifugeranno. [...] Il racconto dà l'impressione di essere stato scritto, per cosi dire, senza levare la penna dai fogli, con una continuità e rapidità da stato di grazia. [...] E questa è la grande, affascinante contraddizione di Candide: che un libro scritto a fondare il pessimismo e a irridere l'ottimismo scorre effettualmente a infondere ottimismo. E almeno in questo senso: che un mondo in cui c'è stato un uomo che ha scritto Candide e in cui ancora ci sono uomini che con uguale spirito lo leggono, è davvero il migliore dei mondi possibili ${ }^{21}$.

Homme de vaste culture, chez qui la citation fait partie intégrante de sa création littéraire, Sciascia se hasarda en 1977, après bien d'autres ${ }^{22}$, à imaginer son propre Candide dans un bref récit intitulé Candido ovvero un sogno fatto in Sicilia. Il a raconté comment son projet s'était peu à peu imposé à lui et quel profit il a tiré de sa réalisation:

Da molto tempo pensavo di scrivere une specie di Candido dei nostri giorni. Non una parodia, ma una reinvenzione sia del personaggio sia di quella velocità e di quella leggerezza che sono nel racconto di Voltaire. [...] Lavorandoci, inventavo l'azione giorno per giorno ed era un gran divertimento non sapere quello che avrei scritto l'indomani. [...] Sapevo cosa doveva entrare nelle esperienze, nelle vicissitudini del personaggio: tutti i miti del tempo, la psicanalisi, il comunismo, ed anche un certo modo di vivere e di intendere il cattolicesimo, il confluire del cattolicesimo nel comunismo e del comunismo nel cattolicesimo. [...] Ė stata per me un'operazione liberatoria, in un certo senso. Perché, nonostante il mio tentativo - sempre - di semplificare, sono anch'io maledettamente complicato ${ }^{23}$.

Quand on sait l'admiration profonde de Sciascia pour l'écrivain Voltaire, ce n'était pas de sa part fausse modestie que de s'inquiéter s'il avait été à la hauteur de son modèle. Peut-être Candide, dit-il dans la note qui suit le roman, a-t-il été récrit cinq ou six cents fois. Quant à lui: «Comunque, che questo mio racconto sia il primo o il seicentesimo, di quella formula ho tentato di servirmi. Ma mi pare di non avercela fatta, e che questo libro somigli agli altri miei. Quella velocità e leggerezza non è più possibile ritrovarle: neppure da me, che credo di non avere mai annoiato il lettore. Se non il risultato, valga dunque l'intenzione: ho cercato di essere veloce, di essere leggero. Ma greve è il nostro tempo, assai greve» (p. 461).

La dernière phrase est une claire allusion au chaos politique et social de l'Italie dans les années soixante-dix et à l'évolution personnelle de Sciascia. Élu conseiller en

(21) L. SCIASCIA, Per un ritratto dello scrittore da giovane, Milano, Adelphi, 2000, pp. 118-119.

(22) Voir J. Vercruysse, Les Enfants de Candide, dans Essays on the Age of Enlightenment in honor of I.O. Wade, Genève, Droz, 1977, pp. 369-376. De
1760 à 1961, on ne compte pas moins de quarantesix contes, comédies, pamphlets, opéras et dialogues inspirés par l'œuvre de Voltaire.

(23) Cité par M. CollurA, Il Maestro di Regalpetra. Vita di Leonardo Sciascia, cit., p. 256. 
janvier 1974 aux élections communales de Palerme sur les listes communistes, Sciascia a rompu avec le Parti en février 1977 et l'écho de cette rupture se retrouve dans son roman, paru en automne de cette année. La rédaction du conte de Voltaire s'était faite elle aussi dans des circonstances agitées - persécutions contre les philosophes, tremblement de terre de Lisbonne, guerre de Sept ans, etc. - qui conduisaient l'auteur à s'en prendre à la philosophie de l'optimisme sous le couvert d'une œuvre de fiction. Comme Voltaire, Sciascia recourt à la fiction pour faire réfléchir à la réalité.

Le héros de Sciascia, Candido Munafò, a vu le jour dans une grotte de Sicile où s'étaient réfugiés ses parents dans la nuit du 9 au 10 juillet 1943, lors du débarquement des troupes américaines et britanniques. D'emblée joue une volontaire prise de distance. Chez Voltaire, le bon sens et la simplicité de l'enfant lui ont valu son nom: «Il avait le jugement assez droit, avec l'esprit le plus simple; c'est, je crois, pour cette raison qu'on le nommait Candide». Rien de tel chez Sciascia, chez qui le héros, fût-il né un jour plus tôt, se serait prénommé Bruno, comme le fils de Mussolini. Le destin voulut donc que ce nom renvoyât au contraire au début d'une ère nouvelle, comme synonyme de page blanche dans l'histoire de son pays libéré du fascisme:

Indicato dal destino - e cioè dagli avvenimenti che da quella sera corsero in Sicilia e in Italia - fu invece il nome che gli misero. [...] Nato dopo il primo e terribile bombardamento della città in cui risiedevano, i genitori gli scelsero invece il nome di Candido. [...] Come una pagina bianca, il nome Candido; sulla quale, cancellato il fascismo, bisognava imprendere a scrivere vita nuova. L'esistenza di un libro intitolato a quel nome, di un personaggio che vagava nelle guerre tra àvari e bulgari, tra gesuiti e regno di Spagna, era perfettamente ignota all'avvocato Francesco Maria Munafò; nonchè l'esistenza di Francesco Maria Arouet, che di quel personaggio era stato creatore. Ed anche alla signora, che qualche libro lo leggeva; a differenza del marito che non uno ne aveva mai letto se non per ragioni di scuola e di professione. Come poi entrambi avessero attraversato ginnasio, liceo e università senza mai sentire parlare di Voltaire e di Candido, non è da stupirsene: capita ancora (pp. 349-350).

Comme le personnage du conte français, Candido fait lui aussi l'apprentissage de la vie, quoique dans des aventures moins lointaines et moins tumultueuses, promenant sur la vie son regard «senza malizia e senza colpa, curioso». Comme par hasard, ou mené par son destin, il débusque ici et là les vices sociaux, les mensonges, les hypocrisies. Ayant surpris une conversation entre son père avocat et un homme qui avoue le crime pour lequel un autre va être puni, n'a-t-il pas, «candidement», révélé la vérité? Son père s'est suicidé pour éviter le déshonneur et, traité par tous de «spia, spione, delatore, traditore» et par sa mère, qui l'abandonne pour suivre un capitaine américain, de «piccolo mostro», Candido est exclu du cercle familial et abandonné aux soins de la gouvernante Concetta. C'est sa première rupture avec une forme de l'autorité.

Candido grandit donc sous la garde de cette femme bigote et superstitieuse, représentation du fanatisme populaire, que condamne l'archiprêtre Antonio Lepanto, le précepteur, esprit ouvert qui se désole de ces catholiques trop nombreux qui «erano ragione della sua preoccupazione di sacerdote; una preoccupazione che a volte arrivava alla disperazione» (p. 379). Don Antonio, prêtre peu banal, est passionné de psychologie, voire de psychanalyse, donc mal vu de l'évêché à qui il a soumis depuis belle lurette un mémoire sur ce sujet scabreux. À la différence de Pangloss, professeur grotesque en perpétuel décalage avec le réel, c'est un homme curieux de tout, qui remet en question ses propres croyances et prend soin de développer l'intelligence critique de Candido. Celui-ci avait été rejeté de sa famille pour avoir dit la vérité; la même mésaventure arrive à don Antonio. À l'occasion d'une enquête sur le meurtre d'un curé, il aide la justice à découvrir le coupable, un avocat qui n'a pu convaincre l'ecclésiastique de défroquer pour épouser sa fille qu'il avait engrossée. Le 
voilà donc au ban de l'Église, regrettablement éclaboussée par ses révélations: «Non che la verità non sia bella: ma a volte fa tanto di quel danno che il tacerla non è colpa ma merito» (p. 391). Rendu à la vie civile, don Antonio emmène son élève à Lourdes, à la fois pour l'immuniser une fois pour toutes contre les mômeries du catholicisme et pour consacrer ses propres adieux à la religion. Candido fera au cours de ce voyage l'expérience vivifiante de la sexualité, mais aussi celle du marchandage éhonté de la foi et de l'espoir, du culte absurde de la Vierge et des saints, au point de conclure: «Se fossi Dio, di tutto questo mi offenderei» (p. 399). Moins heureux que lui, son maître, assurément affranchi de ses liens sacerdotaux, demeure cependant prisonnier de la peur de la femme: «È facile far cadere uno dopo l'altro, come nel baraccone del tiro al bersaglio, tutti i dogmi, i simulacri e i simboli che sono stati parte della tua vita: direi che basta la piccola carabina del Dizionario di Voltaire, se l'occhio è più appannato. Ma tutti quei dogmi, quei simulacri, quei simboli che tu credi di avere abbattuto, vanno a raccogliersi e nascondersi nel corpo della donna, nell'idea dell'amore o semplicemente nel fare all'amore» (p. 402).

Libéré des contraintes de l'ordre familial et de la religion, reste à Candido à se dégager de l'emprise du pouvoir et des idéologies. Son grand-père, le général Cressi, est un ancien fasciste opportunément reconverti en 1948 dans la démocratie chrétienne. Son passé douteux lui a bien valu quelques charges de ses adversaires politiques, mais il les a découragés en menaçant de dévoiler «i nomi, le cariche e le prebende dei fascisti candidati in altre liste, di altri partiti» (p. 370). Surpris de voir le général soutenir des principes contradictoires, l'enfant lui a naïvement demandé: «Hai sbagliato allora o stai sbagliando ora?» - pour s'entendre répondre: «Ma che sbagliare, verme che sei! È la stessa cosa» (p. 382). L'initiation aux déceptions de la politique se poursuit lorsque Candido s'étonne du pouvoir qu'exerce le riche sur le pauvre. Lui-même riche héritier, il se sent mal à l'aise en face des paysans qui travaillent ses terres, mais quand il se met en tête d'y travailler comme eux, il est mal vu de ces simples. Mal vu aussi lorsque, avec don Antonio et lecture faite de Marx, Lénine et Gramsci, il s'inscrit au parti communiste. Sans doute justfie-t-il un peu la suspicion: jeune homme nanti dont la mère a épousé un Américain et dont le grand-père, exgénéral fasciste, a fait peau neuve en démocrate chrétien. Il a cru pourtant au comunisme comme à un substitut de la foi: «Essere comunista era insomma, per Candido, un fatto quasi di natura: il capitalismo portava l'uomo alla dissoluzione, alla fine; l'istinto della conservazione, la volontà di sopravvivere, ecco che avevano trovato forma nel comunismo. Il comunismo era insomma qualcosa che ha che fare con l'amore» (p. 407). Il ne tarde pas à être détrompé. Certes, il aime la compagnie des gens simples, mais observe dans le discours des dirigeants «una ripetizione di quel che nella Chiesa era sempre accaduto ed accadeva» (p. 417). Ce communisme n'est bien qu'une autre religion, non moins fallacieuse que le catholicisme, qui prend l'homme à ses leurres. On prendra pour une extravagance déplacée son désir d'offrir ses terres au peuple et, exclu, il se retrouvera «communista senza partito», tandis que sa famille réussit, par voie judiciaire, à le faire déclarer incapable et à s'approprier ses biens. Après la famille et l'Église, c'est la politique qui chavire dans le jeu de massacre.

Voyager, peut-être? Avec sa cousine Francesca devenue sa compagne, Candido parcourt l'Espagne, la France, l'Égypte, Israël et l'Italie avant de se fixer à Turin. Malgré son expérience sicilienne, Candido a continué, bien que toujours déçu, à hanter des groupes de réflexion communistes, jusqu'au jour où Francesca lui a tenu ce propos de bon sens: «E se fossero soltanto degli imbecilli?» Le moment est donc venu d'oublier le communisme, ce «sogno fatto in Sicilia» et de se fixer à Paris, capitale des lettres, de l'esprit et de la liberté où l'on peut flâner au gré de tous les caprices: 
E una delle ragioni del loro amore a Parigi - oltre quelle dell'amore all'amore, dell'amore alla letteratura, dell'amore alle piccole e vecchie cose e ai piccoli e antichi mestieri - stava nel fatto che vi si poteva ancora camminare, ancora passeggiare, ancora svagatamente andare e fermarsi e guardare. Soltanto a Parigi, per esempio, camminavano tenendosi per mano; soltanto a Parigi il loro passo assumeva una goduta lentezza. Vi si sentivano insomma sciolti e liberi (p. 448).

Cette découverte de la liberté coïncide avec le renoncement à tous les «ismes», aux dogmes, aux théories, aux arcanes de la psychanalyse, aux prétendues révolutions, avec l'élimination des pères encombrants, y compris celui qui enseigne les libertés:

Passarono il ponte Saint-Michel e don Antonio, quasi predicando, cominciò: «Qui, nel 1968, nel mese di maggio...».

«Erano i nostri nonni o i nostri nipoti?» lo interruppe Candido.

«Domanda inquietante» disse don Antonio. E si zitti. Pensava, borbottava.

Dal quai, imboccarono rue de Seine. Davanti alla statua di Voltaire don Antonio si fermò, si afferrò al palo della segnaletica, chinò la testa. Pareva si fosse messo a pregare. «Questo è il nostro padre» - gridò poi - «questo è il nostro vero padre».

Dolcemente ma con forza Candido lo staccò dal palo, lo sorresse, lo trascinò. «Non ricominciamo coi padri» - disse. Si sentiva figlio della fortuna; e felice (pp. 459-460).

On le voit, il serait vain de chercher une correspondance étroite entre Candide et Candido $^{24}$, Sciascia n'ayant nullement souhaité produire une nouvelle mouture du récit voltairien, mais faire référence à un mythe littéraire. Du moins l'un et l'autre héros ont-ils en commun la dénonciation des leurres qui égarent les esprits. Les tribulations de Candide signalaient la présence du mal dans le monde et le mensonge du «Tout est bien», celles de Candido mettent en évidence le fanatisme, la corruption, l'abus d'autorité, l'intolérance. Candide dans sa métairie de la Propontide renonce aux spéculations gratuites pour cultiver son jardin, Candido dissipe à Paris, dans l'exercice vécu de la liberté, le «sogno fatto in Sicilia» - le communisme. L'un et l'autre revendiquent l'action quotidienne et l'autonomie de la pensée. Au terme de ce parcours, contre don Antonio, passé de l'Église au communisme et à mai ' 68 dans le même besoin de référence supérieure, même le «père» Voltaire doit être récusé - «non ricominciamo coi padri». Se libérer même de Voltaire, maître des libertaires, n'est-ce pas en somme appliquer la vraie leçon du «secolo educatore»? Droite ou gauche sont en définitive ennemies du bonheur et du bien-être et, pour le montrer, Sciascia unit le pessimisme sicilien à la causticité voltairienne. On n'échappe aux idéologies qu'en vivant avec les choses et non plus avec les idées ${ }^{25}$. Ce qui lui permet de conclure, à propos de Candido: «In fin dei conti, tutti i miei libri non sono che la storia di una serie di delusioni storiche e personali, presenti e passate, queste viste alla luce del presente, e viceversa ${ }^{26}$.

Sciascia rend ainsi hommage à l'auteur de Candide et du Traité sur la tolérance, sans pour autant sacrifier son indépendance et adapte sa conclusion à l'histoire contemporaine pour faire de son Candido, jusqu'à un certain point, un «anti-Candide» ${ }^{27}$. Comme d'habitude, quelques citations semées ici et là constituent un clin d'œil au

(24) Pour quelques points de rencontre précis ou de «réécriture», voir I.R. MORRISON, Leonardo Sciascia's Candido and Voltaire's Candide, «The Modern Language Review», 2002, 97, 1, pp. 59-71.

(25) A. MotTA, Leonardo Sciascia: la verità, l'as- pra verità, Manduria, Lacaita, 1985, p. 14.

(26) L. SCIASCIA, La Sicilia come metafora, p. 71.

(27) J.-N. Schifano, Sciascia ou de la candeur, «L'Arc», 77, 1979, p. 38. 
lecteur. Sciascia traduit par exemple l'épisode du baiser à Cunégonde qui vaut à Candide d'être chassé par le baron de Thunder-ten-tronckh, mais son héros connaît un sort plus plaisant: «A differenza del suo omonimo, le cui avventure e sventure erano uscite dai torchi del Lambert giusto due secoli prima, Candido ebbe quel giorno un lungo, pieno e quieto godimento. Godimento lungamente, pienamente e quietamente condiviso da Paola. E anche nei giorni che seguirono, nei mesi» (p. 404). Comme dans Candide, les chapitres sont introduits par de brefs résumés où se retrouve le ton voltairien - Della partenza e del ritorno di Amleto; e di quel che meritamente toccò all'avvocato Munafò e immeritamente a Candido - ou l'allusion au récit de référence - Del tentativo che l'ex arciprete feci di dedicarsi a coltivare il proprio orto e Candido le proprie terre; e delle delusioni che ne ebbero. L'humour, l'ironie, la rapidité de la narration font de cette comédie humaine à l'échelle sicilienne ${ }^{28}$ une réécriture subtile et originale, non une imitation, même si Voltaire y affleure partout en filigrane. Si Candido n'est pas une répétition de Candide, ce n'est pas parce que Sciascia aurait été incapable de retrouver la «velocità e leggerezza» de Voltaire, mais parce qu'il a su, avec une suprême habileté, s'affranchir de toute imitation servile sans cesser d'être fidèle à l'esprit du texte et structurer son récit en courts chapitres au rythme narratif rapide.

Par l'art et le style non moins que par les thèmes, les récits de Sciascia, du Consiglio d'Egitto et du Contesto à Todo modo et Candido, portent bien la marque d'un siècle des Lumières synonyme, pour l'écrivain sicilien, de liberté et de progrès, mais à l'égard duquel ses personnages témoignent peu à peu, de Di Blasi et de Rogas à Candido, d'un scepticisme nécessaire, lui-même reflet de l'évolution personnelle de l'auteur et de son détachement des idéologies réductrices. N'est-ce pas Sciascia luimême qui assure: «Lo scetticismo io lo vedo [...] come la valvola di sicurezza della ragione»? ${ }^{29}$.

RAYMOND TROUSSON

(28) J. Dauphiné, Sciascia lecteur de Candide: réception et création, dans Hommage à Jean Richer,
Paris, Les Belles Lettres, 1985, pp. 133-138.

(29) L. Sciascia, La Sicilia come metafora, p. 6. 